\title{
Análise da validade dos parâmetros absolutos e relativos para o cálculo da potência anaeróbia no RAST
}

CDD. 20.ed. 796.07

\author{
Bruno MEZÊNCIO* \\ Rafael SONCIN* \\ João Gustavo de Oliveira CLAUDINO* \\ Jacielle Carolina FERREIRA* \\ Alberto Carlos AMADIO* \\ Júlio Cerca SERRÃO* \\ Rudolf HUEBNER* \\ Leszek Antoni SZMUCHROWSKI*** \\ *Escola de Educação \\ Física e Esporte, \\ Universidade de São \\ Paulo. \\ ** Departamento de \\ Engenharia, Universi- \\ dade Federal de Minas \\ Gerais. \\ *** Escola de Educação \\ Física, Fisioterapia e \\ Terapia Ocupacional, \\ Universidade Federal \\ de Minas Gerais.
}

\begin{abstract}
Resumo
0 objetivo do presente estudo foi analisar a validade dos parâmetros de potência absolutos e relativos do Running-based Anaerobic Sprint Test (RAST) com base no teste de Wingate (WAnT). Doze voluntários do sexo masculino com idade de 25,50 anos $( \pm 2,32)$ realizaram os testes RAST e WAnT. Foram observados valores significativos $(p<0,01)$ de correlação para a potência máxima absoluta $(r=0,76)$ e para a potência média absoluta $(r=0,74)$ dos testes, entretanto, os valores relativos apresentaram correlações não significativas ( $r=0,54$ e 0,27 respectivamente). Todos os parâmetros analisados apresentaram diferenças significativas $(p<0,01)$ na comparação entre WAnT e RAST. Desta forma, os resultados sugerem que apenas os parâmetros de potência absoluta do RAST são válidos. Assim ao aplicar o RAST para avaliação e/ou prescrição de treinamento, ou em pesquisas científicas, devem ser usados preferencialmente os parâmetros absolutos do teste.
\end{abstract}

Palavras-chave: Testes anaeróbios; "Sprints" repetidos; Wingate; Normalização.

\section{Introdução}

A capacidade anaeróbia pode ser entendida como a capacidade do indivíduo de gerar energia através do metabolismo anaeróbio, ou seja, a quantidade total de ATP ressintetizada anaerobicamente ${ }^{1}$ e é um fator determinante do desempenho em diversas modalidades esportivas como futebol ${ }^{2-3}$, handebol ${ }^{4}$, basquetebol ${ }^{5} \mathrm{e}$ ciclismo ${ }^{6}$. Dentre os procedimentos disponíveis para avaliar esta capacidade, Oded Bar-Or e colaboradores propuseram o protocolo de Wingate (WAnT) que consiste em 30 s de esforço máximo em cicloergômetro ${ }^{6-8}$.

Como o WAnT é um protocolo realizado em cicloergômetro, diferentes protocolos foram propostos para se medir o desempenho anaeróbio, por intermédio da utilização de "sprints" repetidos buscando uma maior especificidade, e assim atender as necessidades das modalidades coletivas ${ }^{9}$. Estes protocolos seguem basicamente os mesmos princípios utilizados no WAnT, no qual todos os "sprints" devem ser executados em velocidade máxima e a soma de seus tempos deveria ser de aproximadamente $30 \mathrm{~s}^{10}$. Neste sentido, Draper e White ${ }^{11}$ desenvolveram um protocolo denominado "Running-based Anaerobic Sprint Test" (RAST), que consiste em seis "sprints" máximos de $35 \mathrm{~m}$ com $10 \mathrm{~s}$ de intervalos de recuperação. A potência de cada "sprint" pode ser calculada pela Equação 1. Por meio dela os autores sugerem o cálculo dos parâmetros potência pico, potência média, potência mínima e índice de fadiga.

$$
P=\frac{M \times d^{2}}{t^{3}}
$$

Sendo $M$ a massa corporal do indivíduo, em kg, $d$ à distância, em metros, percorrida em cada "sprint" e $t$ o tempo, em segundos, gasto para percorrê-la. 
Posteriormente, outros estudos passaram a utilizar também o cálculo de valores relativos, normalizados pela massa corporal. Entretanto, a literatura especializada ${ }^{3-4,12-14}$ não aponta de forma clara a justificativa para a adoção de tais parâmetros.

A confiabilidade (teste e re-teste) e validade dos parâmetros do RAST foram verificadas por ZAGATTO et al. ${ }^{14}$. Os parâmetros potência máxima, potência média, potência máxima relativa e potência média relativa apresentaram altos índices de confiabilidade. Para avaliar a validade do RAST os parâmetros absolutos do teste foram comparados com os de WAnT, apresentando correlaçõos elevadas e significativas. No entanto, nada foi reportado a cerca da validade dos parâmetros relativos.

\section{Método}

\section{Amostra}

A amostra foi composta por 12 indivíduos do sexo masculino, $(25,5 \pm 2,3$ anos, $83,14 \pm 11,78 \mathrm{~kg})$ fisicamente ativos. Para evitar influência da especificidade do treinamento nos resultados dos testes, foi adotado como critério de exclusão ser praticante de corrida, esportes coletivos e qualquer modalidade de ciclismo. Todos os voluntários assinaram o termo de consentimento livre e esclarecido. Os procedimentos foram aprovados pelo comitê de ética e pesquisa da Universidade onde o estudo foi realizado (COEP/ UFMG protocolo 0418.0.203.000-9).

\section{Procedimentos}

Previamente à execução dos testes os indivíduos realizaram uma sessão de familiarização para o WAnT e o RAST. Após uma semana foram realizadas duas sessões experimentais com intervalos de aproximadamente 48 horas, em cada sessão foi realizado um dos testes propostos. Tanto na familiarização quanto nas sessōes experimentais a ordem de aplicação dos testes foi aleatorizada para que esta não gerasse interferência nos resultados.

O WAnT foi realizado em um cicloergômetro MAXX (Hidrofit - Brasil) utilizando o programa MCE (JBA - Staniak - Polônia). A execução do WAnT obedeceu aos parâmetros propostos por $\mathrm{BAR}-\mathrm{OR}^{7}$, que estabelece que o indivíduo deve pedalar na maior velocidade possível durante $30 \mathrm{~s}$, com uma carga referente a 7,5\% da sua massa corporal. $\mathrm{O}$ aquecimento curto proposto por INBAR et al. ${ }^{6}$ foi executado previamente ao teste.
A utilização do WAnT como referência para validação no RAST pode ser justificada por dois fatores: a correlação entre o WAnT e corridas de curta duração ${ }^{14-15} \mathrm{e}$ a similaridade da duração dos teste $\mathrm{e}^{14}$. Além disso, outros estudos já realizaram a comparação entre os testes com objetivos diversos ${ }^{13-14,16}$.

Levando em consideração a utilização do RAST como protocolo de avaliação da capacidade anaeróbia e de fatores que a influenciam ${ }^{13,17-18}$, a ampla utilização dos parâmetros relativos do teste e o fato destes não terem sido validados, o objetivo do presente estudo foi verificar a validade dos parâmetros de potência absolutos e relativos do RAST com base no WAnT. Nossa hipótese é de que apenas os parâmetros absolutos do RAST são indicadores da capacidade anaeróbia.

Os parâmetros analisados no WAnT foram: potência máxima (a maior potência gerada durante o teste), potência máxima relativa (a potência máxima normalizada pela massa corporal do avaliado), potência média (média da potência gerada durante os $30 \mathrm{~s}$ do teste) e potência média relativa (a potência média normalizada pela massa corporal do avaliado).

Para execução do RAST foram utilizados dois pares de fotocélulas "multisprint" (Hidrofit - Brasil) e o programa "Multisprint Full" (Hidrofit - Brasil). O sistema permitiu medir o tempo de execução de cada "sprint" e o intervalo de tempo entre os mesmos. Neste experimento, foram realizados seis "sprints" de $35 \mathrm{~m}$ com intervalos de $10 \mathrm{~s}{ }^{11}$. Previamente ao teste, os voluntários realizaram um aquecimento, composto por trote e "sprints" de $10 \mathrm{~m}$, de mesma duração que o aquecimento para o WAnT. O teste foi executado após $3 \mathrm{~min}$. de recuperação.

Os parâmetros analisados no RAST foram: potência máxima (o "sprint" de maior potência), potência máxima relativa (a potência máxima normalizada pela massa corporal do avaliado), potência média (média da potência gerada nos seis "sprints") e potência média relativa (a potência média normalizada pela massa corporal do avaliado).

\section{Análise dos resultados}

A normalidade dos dados foi verificada pelo teste de Kolmogorov Smirnov e a igualdade de variância pelo teste de Levene. Todos os dados apresentaram distribuição normal e igualdade de variância. Para 
comparação dos parâmetros entre os testes foi utilizado um teste $T$ pareado e para avaliar o grau de correlação entre os mesmos foi utilizado o coeficiente de correlação de Pearson. O nível de significância adotado foi de $\mathrm{p}<0,05$ e o programa utilizado foi o SigmaStat 3.5 (Systat Software Inc. - EUA).

\section{Resultados}

A TABELA 1 apresenta os valores de média e desvio padrão de potência máxima (Pmax), potência máxima relativa (Pmaxr), potência média (Pmed) e potência média relativa (Pmedr) do RAST e do WAnT, e os resultados de teste T pareado e Coeficiente de Correlação de Pearson entre os protocolos.

Os parâmetros de potência absoluta e relativa do WAnT foram significativamente maiores aos do RAST. Todos os coeficientes de correlação apresentaram valores positivos, porém os valores das correlaçôes entre os parâmetros absolutos dos testes foram maiores que os apresentados pelos parâmetros relativos.

Os parâmetros potência máxima e potência média apresentaram correlações significativas $(\mathrm{p}<$ 0,05), enquanto para os parâmetros potência máxima relativa e potência média relativa as correlações não foram significativas.

TABELA 1 - Média e desvio padrão dos parâmetros potência máxima (Pmax), potência máxima relativa (Pmaxr), potência média (Pmed) e potência média relativa (Pmedr) nos protocolos de RAST e WAnT, e o valor do coeficiente correlação de Pearson $(r)$.

\begin{tabular}{lccc}
\hline Variáveis & RAST & WAnT & $r$ \\
\hline Pmax (W) & $650,18 \pm 140,79$ & $940,50 \pm 157,44^{*}$ & $0,759^{\#}$ \\
Pmaxr (W/kg) & $7,82 \pm 1,34$ & $11,33 \pm 1,33^{*}$ & 0,514 \\
Pmed (W) & $519,99 \pm 108,69$ & $672,58 \pm 112,33^{*}$ & $0,741^{\#}$ \\
Pmedr (W/kg) & $6,26 \pm 1,02$ & $8,26 \pm 1,49^{*}$ & 0,276 \\
\hline
\end{tabular}

*diferença significativa entre os testes $(p<$ $0,01)$; \# correlação significativa $(p<0,01)$.

\section{Discussão}

O objetivo do estudo foi verificar a validade dos parâmetros absolutos e relativos do RAST. Apenas as potências máxima e média absoluta dos testes apresentaram correlação significativa entre o RAST e o WAnT, enquanto os valores de potência relativa não apresentaram correlação significativa entre os testes. Desta forma, os parâmetros absolutos do RAST são válidos, como já demonstrado por ZAGATTO et al. ${ }^{14}$ que realizaram a mesma comparação entre os testes, encontrando correlações significativas nos parâmetros absolutos, $\operatorname{Pmax}(r=0,53)$ e Pmed $(r=$ $0,46)$, porém neste estudo não foram reportados os resultados para os parâmetros relativos.

Uma possível causa desta discrepância de resultados obtidos para os parâmetros absolutos e relativos do RAST quando comparados ao WAnT pode estar relacionada ao cálculo destas variáveis. No protocolo de WAnT o trabalho realizado em cada giro é constante e o tempo de cada giro é utilizado para determinar a potência ${ }^{6}$. Dessa forma ao se normalizar os resultados pela massa se obtem um índice que indica quanto de potência foi gerada em média por cada quilograma de massa do indivíduo para realizar aquele trabalho constante. Porém, da forma como os parâmetros de potência são calculados no RAST, esta normalização faz com que os resultados passem a ser uma função exclusivamente do tempo, representando assim valores relacionados à velocidade média dos "sprints". Deve-se destacar o fato de a metodologia ter utilizado a corrida e não o cicloergômetro, condição que aumenta a complexidade metodológica dos procedimentos experimentais utilizados.

O WAnT apresentou resultados significativamente maiores que o RAST para todos os parâmetros avaliados. Outros autores também encontraram diferenças estatisticamente significativas na potência máxima e média entre o RAST e WAnT ${ }^{12-14}$. No presente estudo, os voluntários possuíam experiência apenas em treinamento resistido, desta forma é provável que a especificidade dos testes não tenha 
interferido na diferença de desempenho dos voluntários entre os testes. Assim, prováveis causas para a diferença entre os valores dos testes podem estar relacionadas a própria tarefa do teste, diferentes gestos motores sendo executados para medir a mesma capacidade, ou a diferença de massa entre as tarefas, a carga utilizada no WAnT e a massa corporal parta o RAST. Uma segunda possibilidade é que a carga do WAnT foi determinada experimentalmente de forma a maximizar a potência gerada ${ }^{6}$ enquanto a carga do RAST é dependente da massa do voluntário.

Como ambos os protocolos objetivam avaliar a capacidade anaeróbia é esperado que apesar das diferenças significativas entre os resultados, causadas pela diferença entre as tarefas dos mesmos, estes deveriam apresentar correlações positivas e significativas. Porém isso só ocorreu para os parâmetros absolutos dos testes, os mesmos resultados foram encontrados por ZaCharogiannis et al. ${ }^{16}$ e Zagatto et al. ${ }^{14}$. As correlações significativas encontradas para os parâmetros absolutos demonstram com um grau de certeza superior a 99\% que uma maior potência anaeróbica absoluta avaliada no WAnT corresponde a um maior valor também no RAST. Já os parâmetros relativos não apresentaram correlaçõos significativas, isso indica que quando os valores de potência anaeróbica relativa avaliadas pelo WAnT são maiores, a ocorrência de maiores valores no RAST ocorre de forma aleatória em mais de 5\% dos casos. Levando em consideração que ambos os testes são considerados válidos, e que a correlação significativa entre os parâmetros absolutos dos mesmos encontrada também já é reconhecida na literatura, a não associação dos parâmetros relativos indica que alteraçōes na capacidade anaeróbica de um indivíduo pode ser erroneamente interpretada ao se utilizar este parâmetro.

Em conclusão, os parâmetros relativos do RAST podem não representar a real capacidade anaeróbia do indivíduo, assim, ao aplicar o RAST para avaliação e/ou prescrição de treinamento, ou em pesquisas científicas, devem ser usados preferencialmente os parâmetros absolutos do teste.

\begin{abstract}
Analysis of the absolute and relative parameters validity for anaerobic power output in RAST

The aim of the present study was to analyze the absolute and relative parameters validity for Runningbased Anaerobic Sprint Test (RAST) based on Wingate test (WAnT). Twelve male volunteers aged $25.50 \pm$ 2.32 years old performed both RAST and WAnT. Significant $(p<0.01)$ correlation values for the absolute maximum power $(r=0.76)$ and mean absolute power (0.74) was found but the relative mean values did not showed significant correlations for both maximum $(r=0.54)$ and average power output $(r=$ 0.27). Significant differences $(p<0.01)$ between WAnT and RAST were found for all assessed parameters. Therefore, the results of the present study suggest that the RAST should be used with caution for prescription training and evaluation, and acquisition of scientific data. The results also suggest that only the absolute parameters of the test are good predictors of anaerobic power.
\end{abstract}

KEY WORDS: Anaerobic tests; Repeated sprint; Wingate; Normalization.

\title{
Referências
}

1. Reilly T, Bangsbo J, Franks A. Anthropometric and physiological predispositions for elite soccer. J Sports Sci. 2000;18:669-83.

2. Bangsbo J. The physiology of the soccer, with special reference to intense intermittent exercise. Acta Physiol Scand Suppl. 1994;619:1-155.

3. Ribeiro RS, Fúrforo D, Claudino JGO, Gonçalves R. Análise do somatotipo e condicionamento físico entre atletas de futebol de campo sub-20. Motriz. 2007;13:280-7.

4. Roseguini AZ, Silva ASR, Gobatto CA. Determinações e relações dos parâmetros anaeróbios do RAST, do limiar anaeróbio e da resposta lactacidemica obtida no Início, no intervalo e ao final de uma partida oficial de handebol. Rev Bras Med Esporte. 2008;14:46-50. 
5. Balciunas M, Stonkus S, Abrantes C, Sampaio J. Long term effects of different training modalities on power, speed, skill and anaerobic capacity in young male basketball players. J Sports Sci Med. 2006;5:163-70.

6. Inbar O, Bar-Or O, Skinner JS. The Wingate anaerobic test. Champaign: Human Kinetics; 1996.

7. Bar-Or O. Anaerobic performance: mensurement in pediatric exercise science. Champaign: Human Kinetics; 1996.

8. McCormick MC, Baker JS. Considerations in the use of high intensity leg cycle ergometry as a test of muscular performance. Res Sports Med. 2011;19:202-16.

9. Glaister M, Howatson G, Lockey RA, Abraham CS, Goodwin J, McInnes G. Familiarization and reliability of multiple sprint running performance índices. J Strength Cond Res. 2007;21:857-9.

10. Glaister M. Multiple-sprint work: methodological, physiological, and experimental issues. Int J Sports Physiol Perform. 2008;3:107-12.

11. Draper N, White G. New running-based test of anaerobic performance for which you need only a stopwatch and a calculator. Peak Perform. 1997;96:3-5.

12. Buryta R, Skurvydas HA, Novikovas V, et al. Maximal power of football players in preparation period. Sporto Mokslas. 2007;47:78-80.

13. Zagatto AM, Cavalcante WS, Moraes WM. O uso de máscara contra gases na determinação dos testes de velocidade crítica, 12 minutos, wingate e RAST. Rev Educ Fís. 2007;139:4-12.

14. Zagatto AM, Beck WR, Gobatto CA. Validity of the running anaerobic sprint test for assessing anaerobic power and predicting short-distance performances. J Strength Cond Res. 2009;23:1820-7.

15. Patton JF, Duggan A. An evaluation of tests of anaerobic power. Aviat Space Environ Med. 1987;58:237-44.

16. Zacharogiannis E, Paradisis G, Tziortzis S. An evaluation of tests of anaerobic power and capacity. Med Sci Sports Exerc. 2004;36:116.

17. Cipryan L, Gajda V. The influence of aerobic power on repeated anaerobic exercise in junior soccer players. J Hum Kinet. 2011;28:63-71.

18. Gwachan N, Wagner D. Acute effects of a caffeine-taurine energy drink on repeated sprint performance of American College Football Players. Int J Sport Nutr Exerc Metab. 2012;22:109-16.

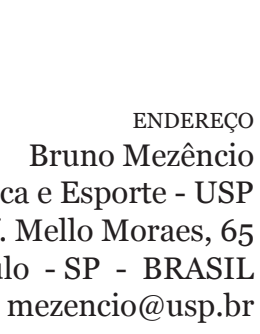

Recebido para publicação: 08/11/2012

1a. Revisão: 06/12/2012

2a. Revisão: 18/12/2012

Aceito: 14/03/2013

Rev Bras Educ Fís Esporte, (São Paulo) 2014 Jan-Mar; 28(1):7-11 • 11 